\title{
Topological and nontopological features of generalized Su-Schrieffer-Heeger models
}

\author{
N. Ahmadi and J. Abouie $\odot^{*}$ \\ Department of Physics, Institute for Advanced Studies in Basic Sciences (IASBS), Zanjan 45137-66731, Iran \\ D. Baeriswyl \\ Department of Physics, University of Fribourg, CH-1700 Fribourg, Switzerland
}

(Received 25 July 2019; accepted 21 April 2020; published 11 May 2020)

\begin{abstract}
The (one-dimensional) Su-Schrieffer-Heeger Hamiltonian, augmented by spin-orbit coupling and longerrange hopping, is studied at half filling for an even number of sites. The ground-state phase diagram depends sensitively on the symmetry of the model. Charge-conjugation (particle-hole) symmetry is conserved if hopping is only allowed between the two sublattices of even and odd sites. In this case, we find a variety of topologically nontrivial phases, characterized by different numbers of edge states (or, equivalently, different quantized Zak phases). The transitions between these phases are clearly signalled by the entanglement entropy. Charge-conjugation symmetry is broken if hopping within the sublattices is admitted. We study specifically next-nearest-neighbor hopping with amplitudes $t_{a}$ and $t_{b}$ for the $A$ and $B$ sublattices, respectively. For $t_{a}=t_{b}$, parity is conserved, and also the quantized Zak phases remain unchanged in the gapped regions of the phase diagram. However, metallic patches appear due to the overlap between conduction and valence bands in some regions of parameter space. The case of alternating next-nearest-neighbor hopping, $t_{a}=-t_{b}$, is also remarkable, as it breaks both charge-conjugation $C$ and parity $P$ but conserves the product $C P$. Both the Zak phase and the entanglement spectrum still provide relevant information, in particular about the broken parity. Thus the Zak phase for small values of $t_{a}$ measures the disparity between bond strengths on $A$ and $B$ sublattices, in close analogy to the proportionality between the Zak phase and the polarization in the case of the related Aubry-André model.
\end{abstract}

DOI: 10.1103/PhysRevB.101.195117

\section{INTRODUCTION}

Since the discovery of quantum Hall effects [1,2], it has been realized that many materials experience different orders in which the topology plays a vital role. Theoretical and experimental investigation of topological states subsequently became an active field of research in different disciplines, such as condensed-matter physics [3,4], photonics [5], and ultracold atomic gases [6]. Recently, much attention has been devoted to the identification and classification of different topological phases of matter. It is quite well known that symmetry and dimension play key roles in the classification of topological properties [7,8]. For one-dimensional (1D) Hermitian systems with chiral symmetry, the topological properties can be characterized by the quantized Berry phase across the first Brillouin zone (Zak phase) [9]. In the absence of chiral symmetry, the Berry phase is not quantized and it is not a topological invariant.

A remarkable quantum-mechanical phenomenon is entanglement. Aside from its notable applications in quantum computation [10], it can be used to probe different phase transitions [11] as well as topological properties of manybody quantum states. For instance, the topological entanglement entropy, the most commonly used measurement of

\footnotetext{
*jahan@iasbs.ac.ir
}

entanglement, is directly related to the total quantum dimension of fractional quasiparticles [12]. Moreover, $\mathrm{Li}$ and Haldane showed that the eigenvalues of the reduced density matrix, called entanglement spectrum (ES), contain complete information about different phases and various phase transitions [13]. The notion of the ES has led to insights in the physics of quantum Hall systems [13-15], delocalizedlocalized phase transition in free fermion models [16], quantum spin systems in one [17-20] and two [21,22] dimensions, topological insulators [23,24], and bilayer lattices [25-27].

One of the most simple topologically nontrivial systems is the SSH model, introduced by Su, Schrieffer, and Heeger in the late '70s [28] to describe both elastic and electronic properties of polyacetylene, a chain of $\mathrm{CH}$ groups. In this model, the elastic and electronic degrees of freedom interact because relative displacements of neighboring $\mathrm{CH}$ groups change the overlap of $\pi$-electron orbitals. For a density of one $\pi$ electron per $\mathrm{CH}$ group, the polymer chain is dimerized in the ground state, i.e., it exhibits an alternating sequence of bond lengths. The dimerization leads to an electronic energy gap between a filled valence band and an empty conduction band. There are two distinct dimerization patterns, and geometric constraints (or doping) can force the coexistence of the two sequences with domain walls separating them. Thus a ring with an odd number of sites has necessarily a domain wall (soliton) in the ground state at half filling. These "intrinsic defects" are also referred to as topological solitons, in view of the origin of their 
stability. Remarkably, a domain wall generates a localized wave function with an energy at midgap. In the context of quantum field theory, the existence of such states due to the coupling of fermions to a classical field with a kinklike spatial dependence had already been discovered before the advent of the SSH model [29]. In quantum chemistry, such states have been proposed even earlier in the form of bond-alternation defects [30]. Experiments on polyacetylene indicate that these solitons play an important role in the electronic and optical properties of the material [31].

In recent years, the electronic part of the SSH Hamiltonian has been frequently used as a toy model for topological insulators. Bond alternation is taken into account by assuming alternating hopping integrals $t(1 \pm \delta)$ with a site-independent dimerization parameter $\delta(|\delta|<1)$. The Hamiltonian reads

$$
H_{\mathrm{SSH}}=t \sum_{i \sigma}\left[(1+\delta) a_{i \sigma}^{\dagger} b_{i \sigma}+(1-\delta) a_{i+1 \sigma}^{\dagger} b_{i \sigma}+\text { H.c. }\right]
$$

where $a_{i, \sigma}^{\dagger}\left(a_{i, \sigma}\right)$ and $b_{i, \sigma}^{\dagger}\left(b_{i, \sigma}\right)$ are fermion creation (annihilation) operators with spin $\sigma$ on the sublattices A and B, respectively, in the unit cell $i$. We have implicitly assumed an even number of sites $(2 L)$. For periodic boundary conditions, the energy spectra for the two dimerization patterns $(\delta<0$ and $\delta>0$ ) are identical, but the eigenstates produce different Berry (or Zak) phases [32]. For open boundary conditions, the two cases have distinct energy spectra. For $\delta<0$, i.e., for two weak bonds at the chain ends, there are two edge states with levels close to midgap. This is the topologically nontrivial phase. For $\delta>0$, i.e., for two strong bonds at the chain ends, there are no edge states.

It is important to notice that the dimerization parameter $\delta$ may be the result of a spontaneous symmetry breaking, as in polyacetylene [31], or it may be produced by external fields, as for cold atoms in optical lattices $[33,34]$. In the latter case, $\delta$ is fixed and cannot be adjusted to lower the energy. However, in the former case, the dimerization pattern can change. In such a situation, the topological phase with two dangling bonds at the chain ends $(\delta<0)$ is unstable because it has a higher energy than the ground-state configuration for $\delta>0$. In this paper, we assume generally $\delta>0$, i.e., we study the case for which the ground state of the SSH Hamiltonian is topologically trivial. Therefore, the topologically nontrivial phases will be produced by extensions of the model.

There are plenty of quasi-one-dimensional conductors, such as the family of organic charge-transfer salts, where the coupling between electrons and elastic degrees of freedom is not produced by the bond-length dependence of the hopping term, but by shifts of local electronic energies due to intramolecular (on-site) displacements (Holstein model [35]). In such a case, the Peierls instability leads to an alternating charge density linked to an energy gap $2 \Delta$. If we again neglect the lattice degrees of freedom, we arrive at the AubryAndré Hamiltonian (for the special case of period-two on-site energies) [36]:

$$
\begin{aligned}
H_{\mathrm{AA}}= & \sum_{i \sigma}\left[t\left(a_{i \sigma}^{\dagger} b_{i \sigma}+a_{i+1 \sigma}^{\dagger} b_{i \sigma}+\text { H.c. }\right)\right. \\
& \left.+\Delta\left(a_{i \sigma}^{\dagger} a_{i \sigma}-b_{i \sigma}^{\dagger} b_{i \sigma}\right)\right] .
\end{aligned}
$$

The Hamiltonians Eqs. (1) and (2) have both different symmetries and different topological properties. However, a simple transformation brings Eq. (2) into the form of Eq. (1), augmented by second- and third-neighbor hopping. This is shown in the Appendix. A combination of Eqs. (1) and (2) leads to the Rice-Mele Hamiltonian [37], which can also be transformed to a generalized SSH model.

Some recent studies have already considered the effects of longer-range hopping. Thus it was found that new phases can be produced by adding next-nearest neighbor hopping to Eq. (1) [38]. There is an important difference between "odd hoppings" (connecting only sites of different sublattices) and "even hoppings" (connecting sites within the sublattices), as they lead to different symmetry classes [39]. In the presence of exclusively odd hoppings, the system belongs to the BDI symmetry class (Altland-Zirnbauer classification $[8,40]$ ), where the topology is characterized by a quantized Berry phase.

Another extension of the Hamiltonian Eq. (1) concerns spin-orbit coupling (SOC), which modifies substantially the band structure and leads to additional topological phases [41-44]. Experimentally, effects of a synthetic SOC on the edge modes of the SSH model have been investigated using a photonic system [45].

In this paper, we consider a generalized SSH model by adding both spin-orbit interaction and longer-range hopping to Eq. (1). In the first part, we treat the case with only odd hopping. Several topological states are found, which can be characterized by quantized Zak phases or, equivalently, by the number of edge states. We also discuss the critical behavior of the topological transitions separating the different phases. The energy levels of the ES, the entanglement gap, and the entanglement entropy correctly reproduce the ground-state phase diagram and help to identify different topological phases. In the second part, we show that the addition of hopping between next-nearest neighbors has drastic effects. Besides putting the system into a different symmetry class, this term strongly affects the phase transitions, the Zak phase, and entanglement properties. Even if the ground states can no longer be labeled by integer winding numbers, interesting relations between the Zak phases and and local quantities appear (polarization for the Aubry-André model, specific correlation functions for the generalized SSH Hamiltonian). We restrict ourselves to specific spatial symmetries, on the one hand, to the case of conserved parity $P$ (where the next-nearest-neighbor hoppings are equal on the two sublattices), on the other hand to the case where parity is not conserved, but the product of charge conjugation $C$ and parity $P$ is (this happens if the next-nearest-neighbor hoppings have the same strengths but opposite signs). Entanglement properties remain relevant also for these "topologically trivial" systems, in particular for the $C P$-symmetric case.

The paper is organized as follows. Section II deals with the chirally symmetric extended SSH model, where even hopping is excluded. The phase diagram of this model is presented in Sec. II A, entanglement is discussed in Sec. II B and the critical behavior is briefly described in Sec. II C. Section III is concerned with the effects of next-nearest-neighbor hopping, which breaks charge-conjugation symmetry. The special cases of $P$ and $C P$ symmetries are analyzed in Secs. III A and III C, 
respectively. The relation between the Zak phase and local physical quantities is elucidated in Sec. III B by means of the polarization in the closely related Aubry-André model. The paper is summarized in Sec. IV.

\section{TOPOLOGICAL PHASES AND ENTANGLEMENT FOR CHIRAL SYMMETRY}

In this section, we study a generalized 1D SSH model with SOC in the presence of odd hopping. The Hamiltonian is

$$
H=H_{\mathrm{SSH}}+H_{\mathrm{SO}}+H_{\mathrm{NNNN}},
$$

where $H_{\mathrm{SSH}}$ is defined by Eq. (1), $H_{\mathrm{SO}}$ is the SOC,

$$
H_{\mathrm{SO}}=\sum_{i \sigma}\left[\lambda a_{i \sigma}^{\dagger} b_{i-\sigma}-\lambda^{\prime} a_{i+1 \sigma}^{\dagger} b_{i-\sigma}+\text { H.c. }\right],
$$

which causes spin flips during hopping, and $H_{\mathrm{NNNN}}$ stands for hopping between third neighbors:

$$
H_{\mathrm{NNNN}}=\sum_{i \sigma}\left[t_{a b} a_{i \sigma}^{\dagger} b_{i+1 \sigma}+t_{b a} b_{i \sigma}^{\dagger} a_{i+2 \sigma}+\text { H.c. }\right] .
$$

We limit ourselves to the case of half filling, where the number of particles is equal to the number of sites.

\section{A. Phase diagram}

For periodic boundary conditions, the Hamiltonian Eq. (3) can be written in momentum space as

$$
H=\sum_{k} \psi_{k}^{\dagger} H_{k} \psi_{k}
$$

where

$$
H_{k}=\left(\begin{array}{cccc}
0 & \varepsilon_{k} & 0 & f_{k} \\
\varepsilon_{k}^{*} & 0 & f_{k}^{*} & 0 \\
0 & f_{k} & 0 & \varepsilon_{k} \\
f_{k}^{*} & 0 & \varepsilon_{k}^{*} & 0
\end{array}\right)
$$

is the so-called Bloch Hamiltonian and $\psi_{k}^{\dagger}=$ $\left(\begin{array}{llll}a_{k \uparrow}^{\dagger} & b_{k \uparrow}^{\dagger} & a_{k \downarrow}^{\dagger} & b_{k \downarrow}^{\dagger}\end{array}\right)$. Here $\varepsilon_{k}=t\left[1+\delta+(1-\delta) e^{-i k}\right]+$ $t_{a b} e^{i k}+t_{b a} e^{-2 i k}, f_{k}=\lambda-\lambda^{\prime} e^{-i k}$, and $\varepsilon_{k}^{*}, f_{k}^{*}$ are complex conjugates of $\varepsilon_{k}, f_{k}$. We choose $t$ as the unit of energy, $t=1$, and assume $0<\delta<1$.

The Hamiltonian Eq. (6) possesses time reversal $(T)$, particle-hole $(C)$, and chiral $(S)$ symmetries and therefore belongs to the BDI symmetry class of symmetry-protected topological (SPT) states $[40,46]$. For the Bloch Hamiltonian Eq. (7), the corresponding operators are $C=\sigma_{0} \otimes \sigma_{z} \kappa, T=$ $\sigma_{x} \kappa \otimes \sigma_{0}$, and $S=T C=\sigma_{x} \otimes \sigma_{z}$, where $\kappa$ produces complex conjugation, $\sigma_{\alpha}$ with $\alpha=x, y, z$ are the Pauli matrices, and $\sigma_{0}$ is the identity matrix. The Bloch Hamiltonian transforms as $[8,46]$

$$
\begin{aligned}
C H(k) C^{-1} & =-H(-k), \quad T H(k) T^{-1}=H(-k), \\
S H(k) S^{-1} & =-H(k) .
\end{aligned}
$$

The matrix Eq. (7) is easily diagonalized and has eigenvalues

$$
E_{1 k}=-E_{2 k}=\left|\varepsilon_{k}+f_{k}\right|, \quad E_{3 k}=-E_{4 k}=\left|\varepsilon_{k}-f_{k}\right|,
$$

symmetric with respect to zero. In general, the spectrum has a gap, but for special parameter values there exist wave vectors for which some energy eigenvalues vanish. This occurs if $\varepsilon_{k}=$ $f_{k}$ or $\varepsilon_{k}=-f_{k}$. We find solutions of these equations for $k=0$ or $k=\pi$ if

$$
t_{a b}=\left\{\begin{array}{l}
-2-t_{b a} \pm\left(\lambda-\lambda^{\prime}\right) \\
2 \delta+t_{b a} \pm\left(\lambda+\lambda^{\prime}\right) .
\end{array}\right.
$$

The gap can also close for other wave vectors provided that $\left|\delta-1+t_{a b} \mp \lambda^{\prime}\right| \leqslant 2\left|t_{b a}\right|$. In this case, the condition for gap closing is given by the quadratic equation:

$$
t_{a b}^{2}-t_{b a}^{2}+\left(\delta-1 \mp \lambda^{\prime}\right) t_{a b}+(\delta+1 \mp \lambda) t_{b a}=0 .
$$

Equations (10) and (11) define the boundaries between different topological phases, which we characterize by the Zak phase [9], the Berry phase [47,48] for Bloch bands,

$$
\Phi=\sum_{E<0} \int_{-\pi}^{\pi}\left\langle u_{k} \mid i \partial_{k} u_{k}\right\rangle d k
$$

where the sum is over the two occupied eigenstates $\left|u_{k}\right\rangle$ of the Bloch Hamiltonian. The Zak phase is not invariant with respect to spatial translations or gauge transformations $[33,34]$, but it can be defined in such a way that its values reflect the number of edge modes $[32,49]$. This bulk-boundary correspondence remains valid if one adds the spin-orbit term. Indeed, for the Hamiltonian $H=H_{\mathrm{SSH}}+H_{\mathrm{SO}}$, one finds two (nontrivial) topological phases, one with $\Phi=\pi$ and one pair of edge modes, the other with $\Phi=2 \pi$ and two pairs of edge modes [43].

The variety of phases increases even more if third-neighbor hopping is added. To calculate the various Zak phases for the full Hamiltonian Eq. (3), we proceed as follows. The eigenvectors of $H_{k}$ for the negative energy eigenvalues are chosen as

$$
\begin{gathered}
\left|u_{k}\right\rangle=\frac{1}{2}\left(\begin{array}{c}
1 \\
-e^{-i \varphi_{k}^{(+)}} \\
1 \\
-e^{-i \varphi_{k}^{(+)}}
\end{array}\right) \quad \text { for } E=-\left|\varepsilon_{k}+f_{k}\right|, \\
\left|u_{k}\right\rangle=\frac{1}{2}\left(\begin{array}{c}
1 \\
-e^{-i \varphi_{k}^{(-)}} \\
-1 \\
e^{-i \varphi_{k}^{(-)}}
\end{array}\right) \quad \text { for } E=-\left|\varepsilon_{k}-f_{k}\right|,
\end{gathered}
$$

where

$$
e^{i \varphi_{k}^{( \pm)}}=\frac{\varepsilon_{k} \pm f_{k}}{\left|\varepsilon_{k} \pm f_{k}\right|} .
$$

The Zak phase is therefore simply

$$
\Phi=\frac{i}{2} \sum_{\tau= \pm 1} \int_{-\pi}^{\pi} d k e^{i \varphi_{k}^{(\tau)}} \partial_{k} e^{-i \varphi_{k}^{(\tau)}}=\frac{1}{2}\left[\varphi_{k}^{(+)}+\varphi_{k}^{(-)}\right]_{-\pi}^{\pi} .
$$

The $2 \pi$ periodicity of $\varepsilon_{k}$ and $f_{k}$ implies that $\varphi_{-\pi}^{(\tau)}$ and $\varphi_{+\pi}^{(\tau)}$ differ by a multiple of $2 \pi$. Therefore the only possible values of the Zak phase are multiples of $\pi$.

We find consistently that for a Zak phase $\Phi=\nu \pi$ there are $|v|$ zero-energy edge modes (bulk-boundary correspondence). The "total winding number" $v$ is well suited for labeling the various phases. In fact, the phase diagram in the $t_{b a}-t_{a b}$ plane, shown in Fig. 1, is a complex patchwork of regions having different values of $v$ (up to $v=I V$ ). The patches are 

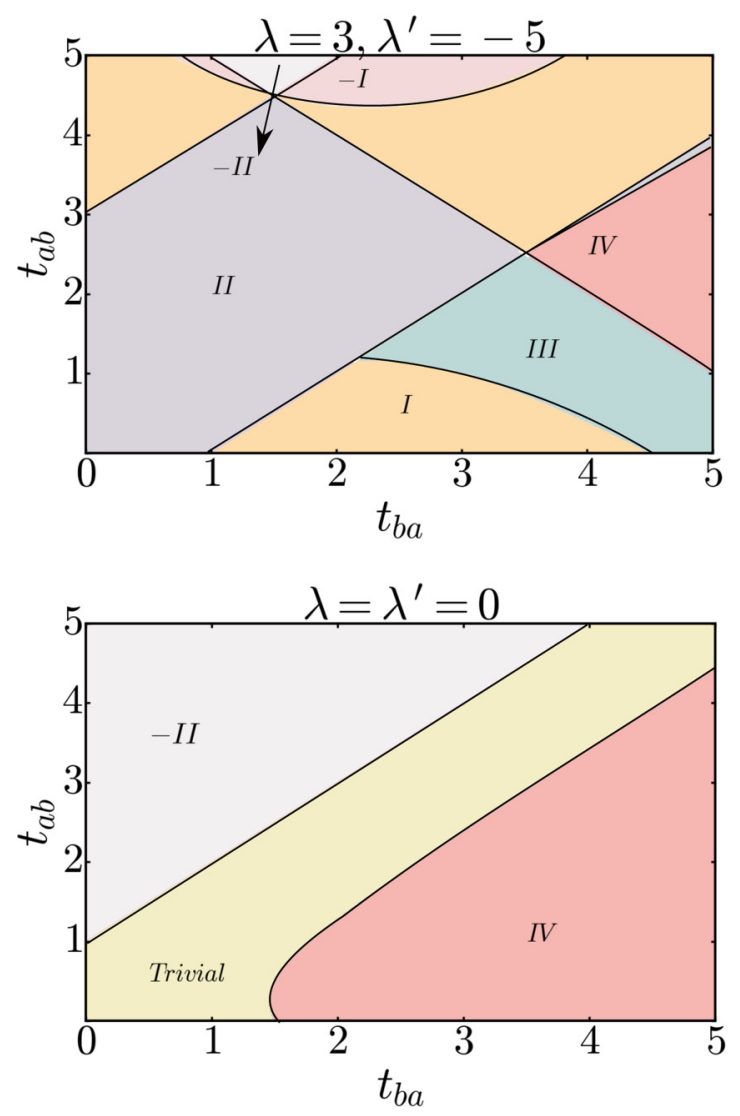

FIG. 1. Ground-state phase diagram of model Eq. (3), for $\delta=$ 0.5 and either finite (top) or vanishing SOC (bottom).

separated from each other either by straight lines (for a gap closing at $k=0$ or $k=\pi$ ) or by curved boundaries (on which the gap closes at $k \neq 0, \pi)$. The transition between two topological phases is always continuous because of the smooth evolution of the band structure as a function of parameters, as exemplified in Fig. 2.

Figure 1 can be qualitatively understood in terms of the "chemical structures" shown in Fig. 3. In the absence of SOC and for weak third-neighbor hopping, we obtain bond
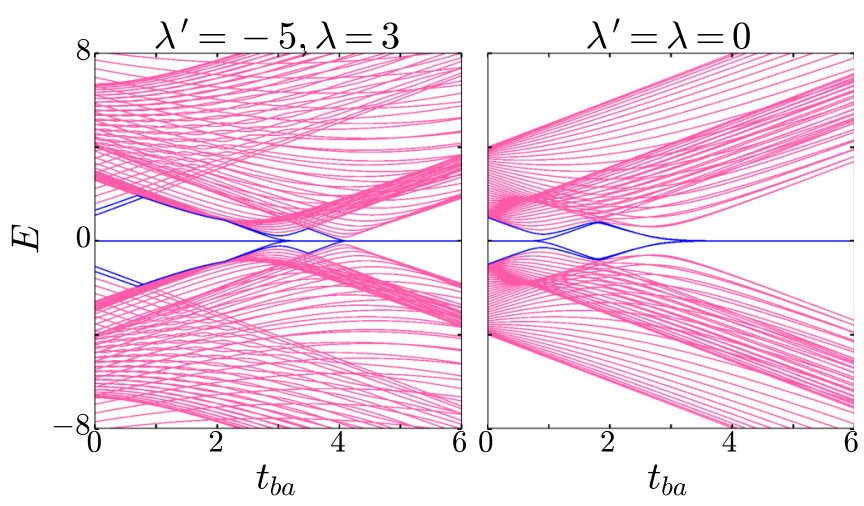

FIG. 2. Band structure of model Eq. (3) for a finite chain of 100 sites (open boundary conditions), and for $\delta=0.5, t_{a b}=2$. The figure on the left refers to a horizontal line in the upper phase diagram of Fig. 1, the one to the right corresponds to the lower phase diagram.

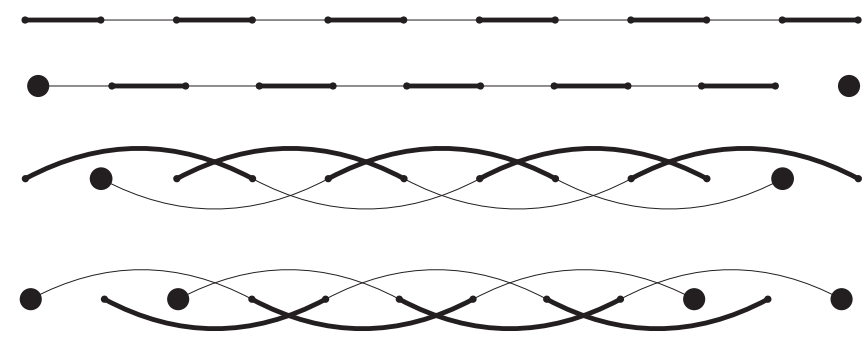

FIG. 3. Bond picture and edge states for a chain with 12 sites. The first two cases represent the bond alternation of the SSH model $\left(t_{a b}, t_{b a} \ll 1\right)$, where the upper (lower) figure has strong (weak) bonds at the edges. The other two cases are for dominant third-neighbor hopping $\left(t_{a b}, t_{b a} \gg 1\right)$ with $t_{a b}>t_{b a}$ (upper figure) and $t_{a b}<t_{b a}$ (lower figure).

alternation without edge states for $\delta>0$ (as assumed here), but two edge modes for $\delta<0$. If third-neighbor hopping dominates, the bonding picture exhibits two edge modes if $t_{a b}$ is the dominant term, but four if $t_{b a}$ is larger, in agreement with Fig. 1.

In Eqs. (13), we have made a particular choice for the phases of the vector components. Instead of choosing the first and third components as real, we could have done so for the second and fourth components. This would give a phase factor $e^{i \varphi_{k}^{( \pm)}}$for the other components, and therefore $\Phi$ would change sign. The phase diagram of course would again be well reproduced in terms of winding numbers and the bulkboundary correspondence would not be affected. However, if we choose an entirely complex representation, with phase factors $e^{i \varphi_{k}^{( \pm)} / 2}$ for the first and third and $e^{-i \varphi_{k}^{( \pm)} / 2}$ for the second and fourth components, the Zak phase vanishes and does not give any information about the phase diagram. Therefore, the "right" gauge choice seems to be a mixed representation. We will see later that the entirely complex representation is the natural choice in cases where the Zak phase is not quantized, but can be related to some local observable.

\section{B. Entanglement}

Entanglement recently became an important tool for characterizing quantum many-body states [50]. To define this concept quantitatively, one divides a system into two parts, a block and an environment, and calculates the reduced density matrix for the degrees of freedom of the block by tracing out those of the environment. In the context of independent (spinless) fermions, a remarkably simple expression has been derived for the reduced density matrix $\rho_{N}$ for a block of $N$ sites in terms of the so-called correlation matrix $G_{N}$ (to be specified below) [51-53]:

$$
\rho_{N}=\operatorname{det}\left(1-G_{N}\right) \exp \left[-\sum_{\ell} \phi_{\ell} c_{\ell}^{\dagger} c_{\ell}\right]=\frac{1}{Z} \exp \left[-H_{\mathrm{ent}}\right] .
$$

Here $H_{\mathrm{ent}}=\sum_{\ell} \phi_{\ell} c_{\ell}^{\dagger} c_{\ell}$ is the so-called entanglement Hamiltonian, and the single-particle ES is given by

$$
\phi_{\ell}=-\left(\ln G_{N}\left(1-G_{N}\right)^{-1}\right)_{\ell \ell}=-\ln \frac{\eta_{\ell}}{1-\eta_{\ell}},
$$

in terms of the eigenvalues $\eta_{\ell}$ of $G_{N}$. 

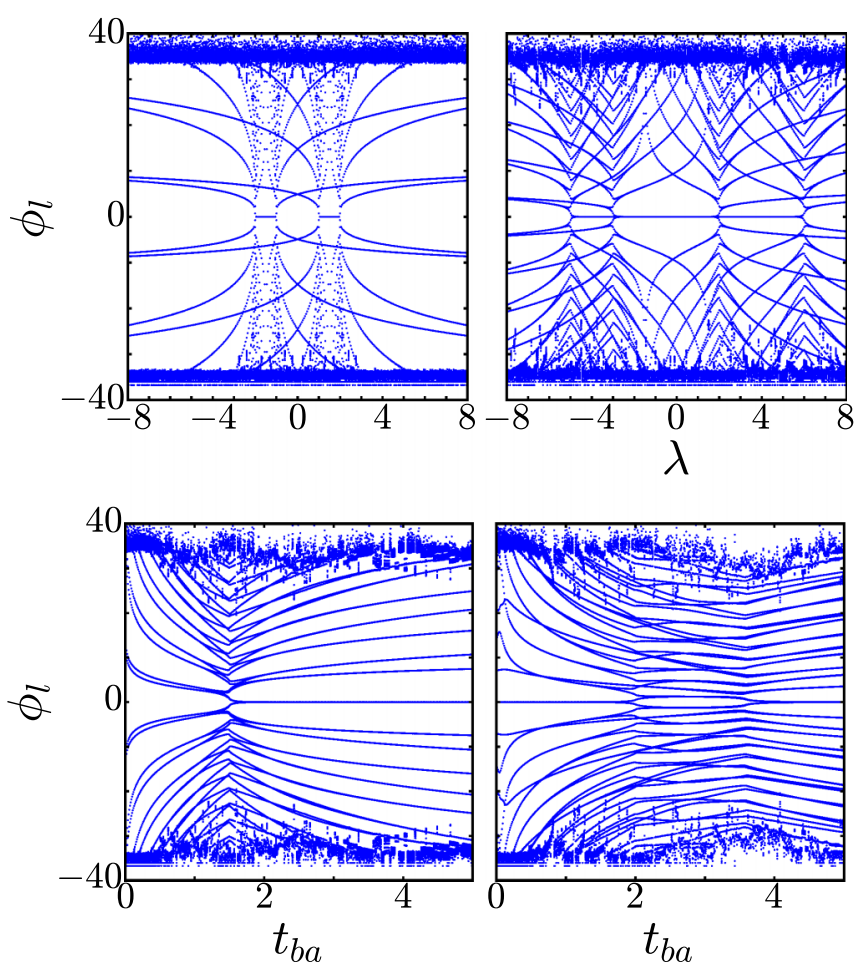

FIG. 4. ES of model Eq. (3), computed for a finite block of 60 sites. Top row: No third-neighbor hopping, $\delta=0.5, \lambda^{\prime}=0$ (left), $\lambda^{\prime}=4$ (right). Bottom row: Vanishing SOC, but finite third-neighbor hopping, $t_{a b}=0.5$ with a critical point at $t_{b a}=1.51$ (left), $t_{a b}=3$ with critical points at $t_{b a}=2$ and 3.59 (right).

In our case, the spin degrees of freedom have to be taken into account explicitly because of the SOC. The correlation matrix $G_{N}$ therefore has $2 N \times 2 N$ matrix elements. At half filling, these are given by the single-particle correlation functions,

$$
\begin{aligned}
\left\langle a_{i \sigma}^{\dagger} a_{j \sigma^{\prime}}\right\rangle & =\left\langle b_{i \sigma}^{\dagger} b_{j \sigma^{\prime}}\right\rangle=\frac{1}{2} \delta_{i j} \delta_{\sigma \sigma^{\prime}}, \\
\left\langle a_{i \sigma}^{\dagger} b_{j \sigma}\right\rangle & =\frac{-1}{8 \pi} \int_{-\pi}^{\pi} d k e^{-i k d}\left(\frac{1}{E_{k}^{-}}+\frac{1}{E_{k}^{+}}\right), \\
\left\langle a_{i \sigma}^{\dagger} b_{j-\sigma}\right\rangle & =\frac{1}{8 \pi} \int_{-\pi}^{\pi} d k e^{-i k d}\left(\frac{1}{E_{k}^{-}}-\frac{1}{E_{k}^{+}}\right), \\
\left\langle b_{i \sigma}^{\dagger} a_{j \sigma}\right\rangle & =\frac{-1}{8 \pi} \int_{-\pi}^{\pi} d k e^{-i k d}\left(E_{k}^{-}+E_{k}^{+}\right), \\
\left\langle b_{i \sigma}^{\dagger} a_{j-\sigma}\right\rangle & =\frac{1}{8 \pi} \int_{-\pi}^{\pi} d k e^{-i k d}\left(E_{k}^{-}-E_{k}^{+}\right),
\end{aligned}
$$

where $E_{k}^{ \pm}=\left|\varepsilon_{k} \pm f_{k}\right|$ and $d=|i-j|$. By computing these integrals, we can obtain the ES of the Hamiltonian Eq. (3) from Eq. (17).

Figure 4 shows the ES of the extended SSH model with and without third-neighbor hopping. Different phases correspond to different patterns of the ES, and critical points occur where these patterns change drastically. Particle-hole symmetry is also manifest. An interesting observation on the zero modes is worth mentioning. They appear at the same parameter values
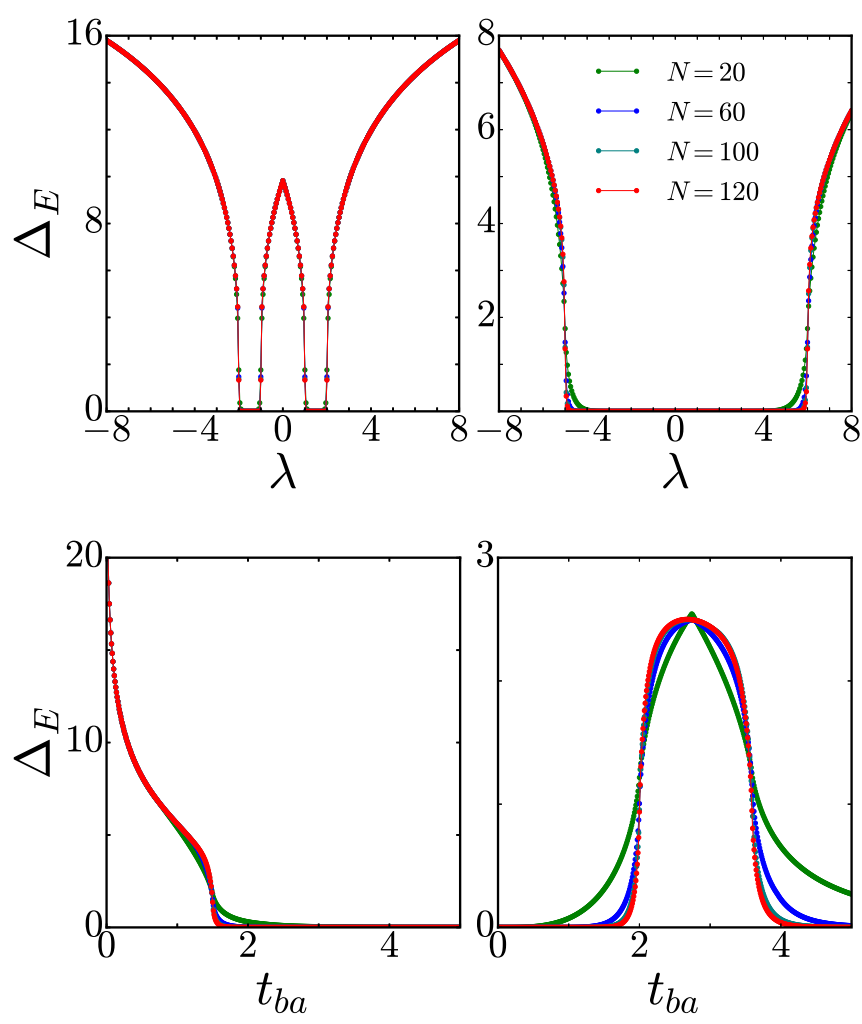

FIG. 5. Entanglement gap for $\delta=0.5$ and different subsystem sizes. Top: No third-neighbor hopping, $\lambda^{\prime}=0$ (4) in the left (right) plot. Bottom: Finite third-neighbor hopping, $\lambda=\lambda^{\prime}=0$ for $t_{a b}=0.5$ (3), in the left (right) plot.

in the ES as in the band structure of the full Hamiltonian, and the numbers of zero modes are identical.

The ES can give a qualitative picture of the various phases. To gain more accurate information, for instance about the critical points, one has to consider quantities which are more specific. One of them is the entanglement gap, defined as the difference between the lowest positive level and the highest negative level as [54]

$$
\Delta_{E}=\left|\phi_{\ell}^{+}-\phi_{\ell}^{-}\right| .
$$

Figure 5 shows the entanglement gap as a function of both SOC and third-neighbor hopping. The gap is finite in topologically trivial phases (with no zero modes) and vanishes in nontrivial phases. To see a sharp onset of a finite gap, one has to choose large subsystem sizes.

Another useful quantity is the entanglement entropy $S$, which can be defined in terms of the eigenvalues of the correlation matrix $G_{N}$,

$$
S=-\sum_{\ell} \eta_{\ell} \ln \eta_{\ell}-\sum_{\ell}\left(1-\eta_{\ell}\right) \ln \left(1-\eta_{\ell}\right) .
$$

Eigenvalues close to 0 or 1 have little weight, and $S$ is dominated by a small interval around $\eta=\frac{1}{2}$. Away from critical points, the spectrum of the correlation matrix is gapped, eigenvalues are close to either 0 or 1 , and $S$ is small. Close to a critical point, a significant part of the eigenvalues is clustered around $1 / 2$, and $S$ diverges at criticality for $N \rightarrow \infty$ (see Fig. 6). This will be further discussed in the next subsection. 

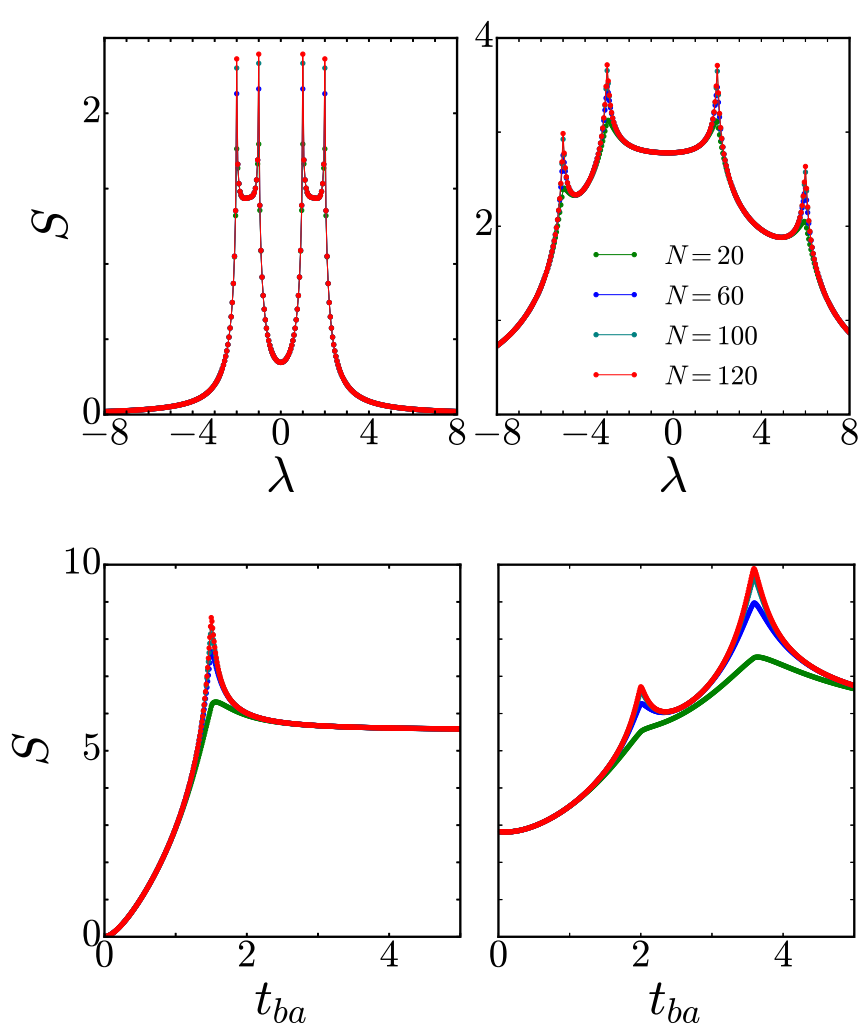

FIG. 6. Entanglement entropy for $\delta t=0.5$ and different subsystem sizes. Top: No third-neighbor hopping, $\lambda^{\prime}=0$ (4) in the left (right) plot. Bottom: Finite third-neighbor hopping, $\lambda=\lambda^{\prime}=0$, $t_{a b}=0.5$ (3), in the left (right) plot.

By analyzing the eigenvalues of the correlation matrix $G_{N}$, we realize that in the topologically trivial phase all eigenvalues of $G_{N}$ are localized in narrow intervals around zero and one, while in SPT-II a non-negligible portion of eigenvalues is clustered around 0.5. By contrast, in SPT-I the distribution of eigenvalues around 0 and 1 is wider. So, it is clear that $S_{\mathrm{II}}>S_{\mathrm{I}}>S_{\text {trivial }}$ (see also Fig. 6). On the other hand, $\Phi_{\mathrm{II}}>\Phi_{\mathrm{I}}>\Phi_{\text {trivial }}$. These two relations indicate that the larger the Berry phase $\Phi$, the larger the entanglement entropy $S$, a possible consequence of the bulk-edge correspondence [55].

\section{Critical behavior}

The lack of an order parameter at a topological phase transition requires alternative quantities to be considered. The fidelity susceptibility has been widely used [56-59]; it represents energy fluctuations in the ground state and thus is something like a quantum specific heat. Another important quantity is the correlation length $\xi$, which has been extracted from the penetration of edge states into the bulk [60]. A different scheme for calculating $\xi$ has been proposed in terms of the Berry connection $\left(\left\langle u_{k} \mid i \partial_{k} u_{k}\right\rangle\right)$ in one dimension and the Berry curvature in two dimensions; their Fourier transforms can be interpreted as real-space correlation functions involving the electric polarization and orbital currents in one and two dimensions, respectively $[61,62]$.
The distinction between conventional quantum phase transitions (with symmetry breaking) and topological transitions is not always clear-cut. As discussed in Sec. I, the SSH Hamiltonian Eq. (1) exhibits a topological transition at $\delta=0$ from a topologically trivial to a topologically nontrivial phase. At the same time, this transition can also be understood in terms of symmetry breaking. In fact, translational symmetry is broken by $\delta \neq 0$, leading to bond alternation with different sequences for different signs of $\delta$. For the generalized SSH Hamiltonian Eq. (3), the phase diagram exhibits a variety of phases, characterized by different winding numbers, as illustrated in Fig. 1. These phases in general do not differ with respect to symmetry and therefore the transitions are of genuine topological nature. Fortunately, in the present case it is very simple to determine the critical behavior, which depends only on the single-particle spectrum close to the $k$ point where the gap closes at criticality [60].

Along the critical lines of Fig. 1, the low-energy singleparticle spectrum has in general a linear dispersion and the critical behavior should therefore be that of a conformal field theory with central charge $c=1$ [63]. This fundamental quantity, which is "(almost) sufficient to characterize a critical model" [64], can be calculated from the entanglement entropy $S$. It is known that at criticality the entanglement entropy of a block of size $N$ scales as $S(N)=\frac{c}{3} \ln N+S_{0}$, where $S_{0}$ is a nonuniversal constant [65]. We have calculated $S$ as a function of block size $N$ in the absence of third-neighbor hopping, $t_{a b}=t_{b a}=0$, for various critical points. Along the critical lines, we consistently find $c=1$, as expected. But what happens at the crossing of two such lines, for instance for $\delta=0.5, \lambda=0.5, \lambda^{\prime}=-1.5$, where four phases meet, two topological phases with $v=\mathrm{I}$, one with $v=\mathrm{II}$ and a trivial phase? The low-energy spectrum has two Dirac cones, one at $k=0$, the other at $k=\pi$. Therefore we get two fields and a central charge $c=2$. This is indeed what we also obtain from the entanglement entropy.

\section{EFFECTS OF BROKEN CHARGE-CONJUGATION SYMMETRY}

So far, we have limited ourselves to systems with hopping between different sublattices ("odd hopping") and therefore with charge-conjugation (particle-hole) symmetry. In this section we investigate the effect of next-nearest-neighbor hopping, which breaks this symmetry. The Hamiltonian is

$$
H=H_{\mathrm{SSH}}+H_{\mathrm{SO}}+H_{\mathrm{NNN}}+H_{\mathrm{NNNN}},
$$

where $H_{\mathrm{SSH}}$ and $H_{\mathrm{NNNN}}$ are defined by Eqs. (1) and (5), respectively, $H_{\mathrm{SO}}$ is the SOC Eq. (4) and $H_{\mathrm{NNN}}$ is the additional hopping term, connecting sites of the same sublattice,

$$
H_{\mathrm{NNN}}=\sum_{i \sigma}\left(t_{a} a_{i \sigma}^{\dagger} a_{i+1 \sigma}+t_{b} b_{i \sigma}^{\dagger} b_{i+1 \sigma}+\text { H.c. }\right) \text {. }
$$

The addition of $H_{\mathrm{NNN}}$ destroys the symmetry between positive- and negative-energy levels, or between conduction and valence bands. As shown in Fig. 7, the band gap, i.e., the spacing between the conduction-band minimum and the valence-band maximum, decreases as $t_{a}$ and $t_{b}$ increase, and it closes on a critical line, which signals a transition from an insulating to a (semi) metallic phase. The interesting region 


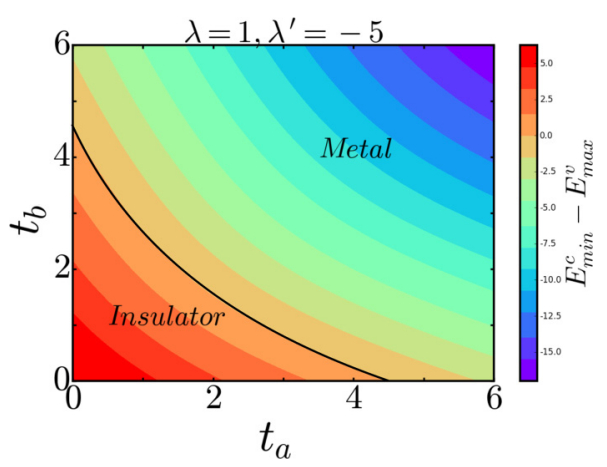

FIG. 7. Band gap as a function of the hopping amplitudes $t_{a}$ and $t_{b}$ for $\delta=0.5, \lambda=1, \lambda^{\prime}=-5$ and $t_{a b}=t_{b a}=0$. The (black) solid line marks the boundary where the band gap vanishes. This is a transition line between insulating and metallic phases.

for our study is the insulating phase where the spectrum is gapped at the Fermi energy. Therefore, the hopping amplitudes between next-nearest neighbors should not be too large.

In the presence of second-neighbor hopping, time-reversal symmetry $T$ is preserved, but this is not the case for charge conjugation $C$ nor for the chiral symmetry $S$. Therefore, the Hamiltonian Eq. (21) belongs to the AI symmetry class. According to the periodic table of topological insulators/superconductors [40,66,67], this class is topologically trivial. Nevertheless, some properties resemble features found in topological phases, especially if the hopping parameters $t_{a}$ and $t_{b}$ are chosen in such a way that the system keeps a spatial symmetry. We consider the cases $t_{a}=t_{b}$, where parity $P$ is conserved and $t_{a}=-t_{b}$, where the product $C P$ is conserved, while individually both $C$ and $P$ are broken.

\section{A. Conserved parity}

Figure 8 illustrates the connectivity induced by nearestand next-nearest-neighbor hopping, and it shows how a given pattern changes due to a parity operation, where $b$ sites at $i$ are exchanged with $a$ sites at $L+1-i, i=1, \ldots, L$. Clearly, the pattern is invariant if $t_{a}=t_{b}$. For the Bloch Hamiltonian $H_{k}$, which is again a $4 \times 4$ matrix as in Eq. (7), with additional elements $2 t_{a} \cos k$ and $2 t_{b} \cos k$ on the diagonal, parity corresponds to the operator $i \kappa \sigma_{0} \otimes \sigma_{x}$. One finds $P H_{k} P^{-1}=H_{k}$, provided that $t_{a}=t_{b}$.
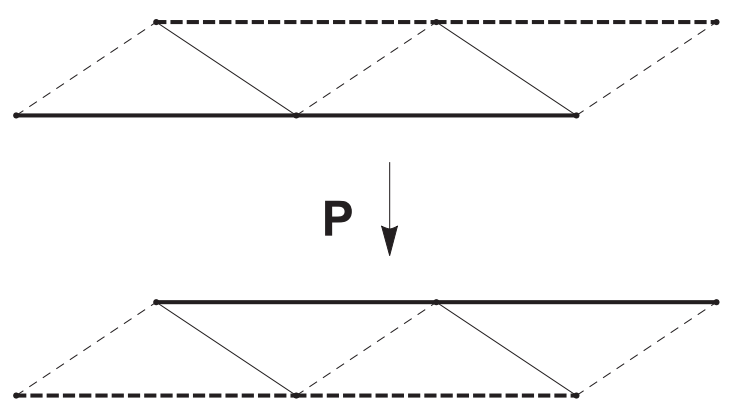

FIG. 8. Effect of the parity operation on a chain with both nearest-neighbor (thin lines) and next-nearest-neighbor bonds (thick lines). The initial configuration is restored if the second-neighbor hopping amplitudes are equal in the two sublattices. Third-neighbor hopping (not shown) does not change the argument.

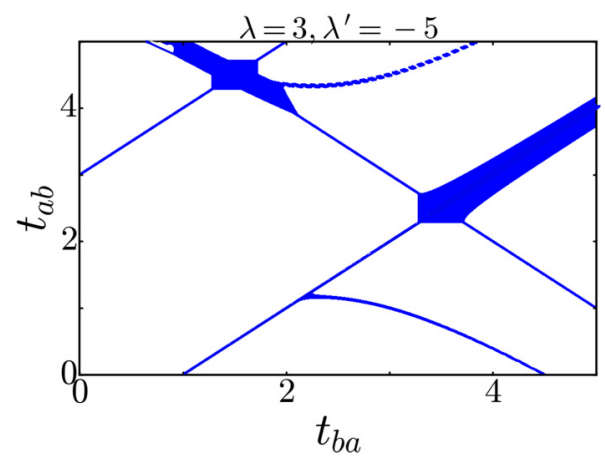

FIG. 9. Phase diagram for the same parameters as in Fig. 1 plus NNN hopping with $t_{a}=t_{b}=0.1$. Metallic regions are shaded.

The eigenvalues of the Bloch Hamiltonian are $2 t_{a} \cos k+$ $\left|\varepsilon_{k} \pm f_{k}\right|$ ("conduction bands") and $2 t_{a} \cos k-\left|\varepsilon_{k} \pm f_{k}\right|$ ("valence bands"). The phase diagram is determined by calculating the difference between conduction band minima and valence band maxima. For finite $t_{a}$, this difference not only can vanish ("gap closing") but it can be negative, as already illustrated in Fig. 7. Even for weak NNN hopping, this can happen in the vicinity of transition lines, which may broaden into metallic patches. In fact, some of the topological transitions found for $t_{a}=0$ are split and replaced by pairs of metal-insulator transitions, as shown in Fig. 9.

Interestingly, in the gapped regions of the phase diagram, where valence and conduction bands are separated, the Zak phase remains quantized even in the presence of NNN hopping. Indeed, for the lowest two eigenvalues $2 t_{a} \cos k-\mid \varepsilon_{k} \pm$ $f_{k} \mid$ the eigenstates $\left|u_{k}\right\rangle$ satisfy exactly the same equations as for $t_{a}=0$, and therefore the Zak phase is not affected.

Some energy spectra and edge states of the Hamiltonian Eq. (21) with $t_{a}=t_{b}$ are represented in Fig. 10 for different values of $t_{a}$ (and $t_{a b}=t_{b a}=0$ ). There is an interesting symmetry between energy levels. To each energy eigenvalue $E$ for a given value of $t_{a}$ there exists an eigenvalue $-E$ if $t_{a}$ is replaced by $-t_{a}$. This is easily understood as follows. The simple transformation $a_{i \sigma} \rightarrow-a_{i \sigma}, b_{i \sigma} \rightarrow b_{i \sigma}$ changes the signs of both $H_{\mathrm{SSH}}$ and $H_{\mathrm{SO}}$, but leaves $H_{\mathrm{NNN}}$ invariant. If, however, at the same time $t_{a}$ is replaced by $-t_{a}$, also $H_{\mathrm{NNN}}$ changes sign.

To investigate the stability of the edge states, we have added a perturbation of the form

$$
H_{p}=\sum_{i \sigma} U_{\sigma}\left(a_{i \sigma}^{\dagger} a_{i \sigma}+b_{i \sigma}^{\dagger} b_{i \sigma}\right)
$$

This term breaks parity. We found that its addition quickly eliminates the edge states, which therefore appear to be "protected by parity". However, in contrast to chiral symmetry, which protects both the energy and the number of edge states (as long as the band gap is finite), this is no longer true in the present case. Figure 10 exemplifies that for finite NNN hopping the levels of edge states move and even may merge with band states before the band gap closes. 

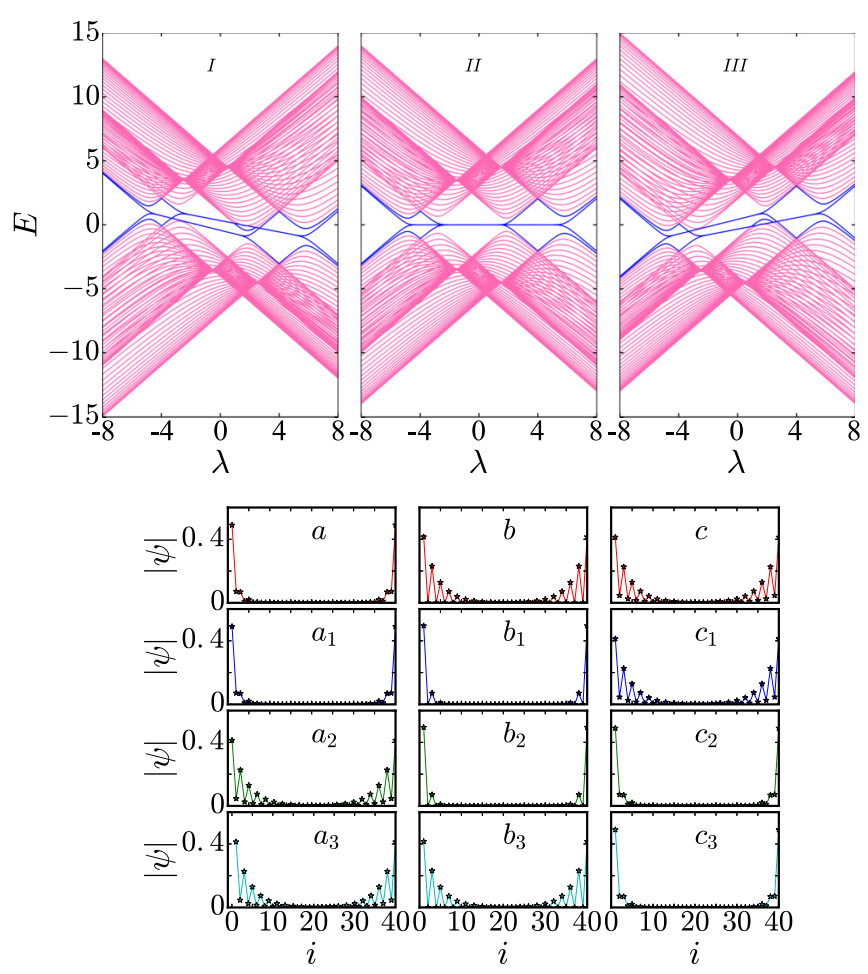

FIG. 10. Top: Energy spectrum of a chain with $L=60$ sites versus $\lambda$, for $\lambda^{\prime}=4$ and different NNN hopping strengths: (I) $t_{a}=t_{b}=$ -0.5 , (II) $t_{a}=t_{b}=0$, and (III) $t_{a}=t_{b}=0.5$. Bottom: Probability amplitude for edge states along a chain of size $L=40$, for $\lambda=-1$ and $\lambda^{\prime}=4$. The energies of the edge states are $E=0.55$ and -0.142 , respectively, for the upper and lower plots on the left, $E=0.142$ and -0.55 , respectively, for the upper and lower plots on the right, and $E=0$ for the plots in the middle.

\section{B. Broken parity, $C P$ invariance, and a physical interpretation of the Zak phase}

As an interlude, we consider the Hamiltonian $H_{\mathrm{AA}}$, Eq. (2), for which both parity $P$ and charge conjugation $C$ are broken. However, as illustrated in Fig. 11, a parity operation followed by charge conjugation restores the original alternating potential. The model is $C P$ invariant.

In Fourier space, the Hamiltonian reads

$$
H_{\mathrm{AA}}=\sum_{k \sigma} \psi_{k \sigma}^{\dagger} H_{k} \psi_{k \sigma}
$$

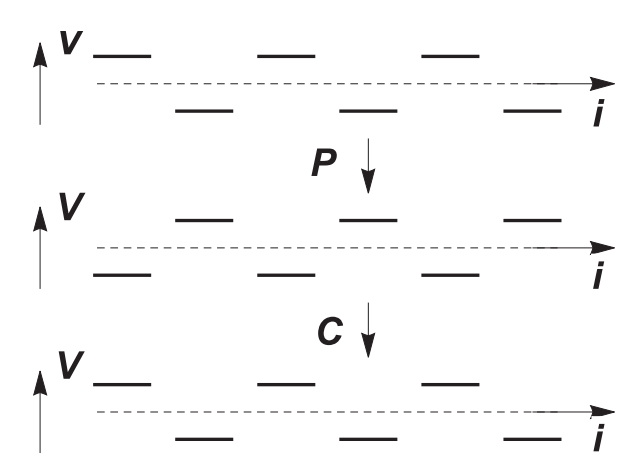

FIG. 11. $C P$ symmetry of the alternating on-site potential. The $C$ and $P$ operations are up-down and right-left reflections, respectively. where $\psi_{k \sigma}^{\dagger}=\left(a_{k \sigma}^{\dagger}, b_{k \sigma}^{\dagger}\right)$ and

$$
H_{k}=\left(\begin{array}{cc}
\Delta & 1+e^{-i k} \\
1+e^{i k} & -\Delta
\end{array}\right)
$$

The eigenvalues are $\pm E_{k}$, where

$$
E_{k}=\sqrt{4 \cos ^{2} \frac{k}{2}+\Delta^{2}}
$$

At half filling, the system is insulating, with a gap $2 \Delta$ separating valence and conduction bands. Normalized eigenstates of $H_{k}$ for negative eigenvalues $-E_{k}$ are

$$
\left(\begin{array}{l}
u_{k}^{(1)} \\
u_{k}^{(2)}
\end{array}\right)=\frac{1}{\sqrt{2 E_{k}}}\left(\begin{array}{l}
\sqrt{E_{k}-\Delta} e^{-i \frac{k}{4}} \\
-\sqrt{E_{k}+\Delta} e^{i \frac{k}{4}}
\end{array}\right) .
$$

The calculation of the Zak phase is greatly facilitated by the fact that the derivative of an even function is an odd function. Therefore, only the derivatives of the phase factors $e^{ \pm i k / 4}$ give finite contributions to the integral in Eq. (12). We obtain (a factor of 2 comes from the spin)

$$
\Phi=2 i \sum_{\nu=1}^{2} \int_{-\pi}^{\pi} d k u_{k}^{(\nu) *} \partial_{k} u_{k}^{(v)}=-\frac{1}{2} \int_{-\pi}^{\pi} d k \frac{\Delta}{E_{k}} .
$$

This expression can be written explicitly in terms of the elliptic integral $K$, but the important points are, on the one hand, that $\Phi$ is not quantized, in contrast to the case of the SSH Hamiltonian, on the other hand, that $\Phi$ is proportional to the charge polarization $P_{\mathrm{ch}}$, as will be demonstrated now.

The Hamiltonian Eq. (24) is diagonalized by the Bogolyubov transformation

$$
\begin{aligned}
& a_{k \sigma}=\cos \vartheta_{k} \alpha_{k \sigma}+e^{-i \frac{k}{2}} \sin \vartheta_{k} \beta_{k \sigma}, \\
& b_{k \sigma}=-e^{i \frac{k}{2}} \sin \vartheta_{k} \alpha_{k \sigma}+\cos \vartheta_{k} \beta_{k \sigma} .
\end{aligned}
$$

With the choice

$$
\tan 2 \vartheta_{k}=-\frac{2}{\Delta} \cos \frac{k}{2}, \quad \cos 2 \vartheta_{k}=\frac{\Delta}{E_{k}},
$$

the transformed Hamiltonian is

$$
H_{\mathrm{AA}}=\sum_{k \sigma} E_{k}\left(\alpha_{k \sigma}^{\dagger} \alpha_{k \sigma}-\beta_{k \sigma}^{\dagger} \beta_{k \sigma}\right) \text {. }
$$

It is now straightforward to calculate the particle densities on the two sublattices and therefore also the polarization

$$
P_{\mathrm{ch}}:=\left\langle b_{i \sigma}^{\dagger} b_{i \sigma}\right\rangle-\left\langle a_{i \sigma}^{\dagger} a_{i \sigma}\right\rangle \text {. }
$$

With $\left\langle\beta_{k \sigma}^{\dagger} \beta_{k \sigma}\right\rangle=1$ and $\left\langle\alpha_{k \sigma}^{\dagger} \alpha_{k \sigma}\right\rangle=0$, we obtain (in the thermodynamic limit)

$$
P_{\mathrm{ch}}=\frac{1}{2 \pi} \int_{-\pi}^{\pi} d k \frac{\Delta}{E_{k}}=-\frac{\Phi}{\pi} .
$$

The amazing proportionality between the Zak phase $\Phi$ and the polarization $P_{\mathrm{ch}}$ is the basis of the modern theory of polarization [68,69]. It shows that the Zak phase can have a deep physical meaning even when it is not quantized. We notice that the proportionality Eq. (32) only holds for the complex representation chosen in Eq. (27), for which $\Phi$ vanishes in the limit $\Delta \rightarrow 0$ 

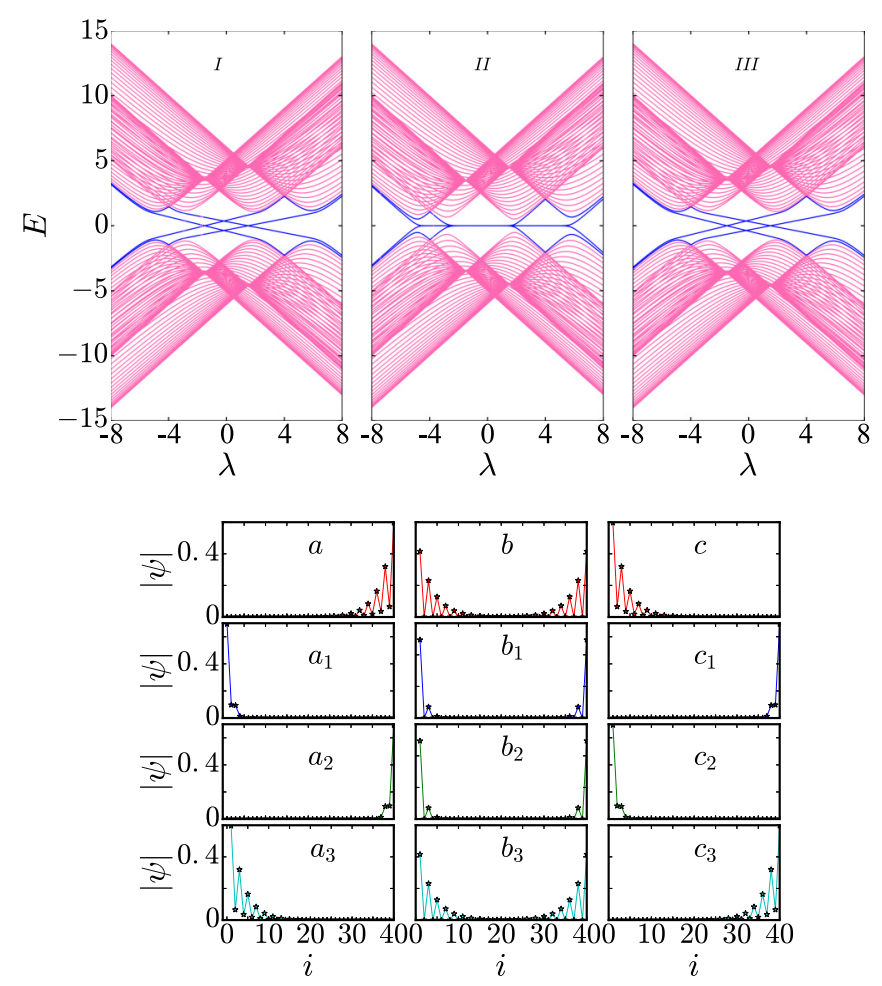

FIG. 12. Top row: The energy spectrum of a chain with $L=60$ sites versus $\lambda$, at $\lambda^{\prime}=4$, and different strengths of second $\mathrm{NN}$ hoppings: (I) $t_{a}=-t_{b}=-0.5$, (II) $t_{a}=t_{b}=0$, and (III) $t_{a}=-t_{b}=$ 0.5 . Bottom row: The probability amplitude of the edge states versus the system size $\mathrm{L}$, at $\lambda=-1$ related to the energies $E=-0.54$, $-0.13,0.13$, and 0.54 , from up to down in left and right plots, and $E=0$ for the middle plot.

\section{C. $C P$ symmetry for $t_{a}=-t_{b}$}

We now return to the generalized SSH Hamiltonian Eq. (21) and concentrate on the case $t_{a}=-t_{b}$ and $t_{a b}=t_{b a}=$ 0 . This is the Aubry-André model in disguise (as shown in the Appendix), with $C P$ symmetry. In fact, one readily shows that $C P$ acts on the Bloch Hamiltonian $H_{k}$ as $C P=\sigma_{0} \otimes$ $\sigma_{y}$, and that $C P H_{k}^{*}(C P)^{-1}=-H_{k}$, if $t_{a}=-t_{b}$. Such a system has two topological-insulator phases, "protected by $C P$ symmetry" [70].

Energy spectra and associated edge states for the $C P$ symmetric case $\left(t_{a}=-t_{b}\right)$ are shown in Fig. 12 for $t_{a}=-0.5$ (left) and $t_{a}=+0.5$ (right), in comparison to the case of chiral symmetry $\left(t_{a}=0\right.$, middle). The results differ from those of the parity-symmetric case (Fig. 10) in two respects. On the one hand, in Fig. 12 the spectra have particle-hole symmetry (not so in Fig. 10), on the other hand, the edge states of Fig. 12 (for $t_{a} \neq 0$ ) are not reflection symmetric, but rather concentrated on the left or right end of the chain (while they are symmetric in Fig. 10). Edge states no longer have zero energy for $t_{a} \neq 0$ because the chiral symmetry, which protects the zero modes for $t_{a}=t_{b}=0$, is broken. Nevertheless, the $C P$ symmetry protects the edge modes to some extent because if it is broken these states quickly disappear. We have verified this behavior explicitly by adding the perturbation Eq. (23), which breaks the $C P$ symmetry.

An interesting aspect of Fig. 12 is that, while the level spectrum is independent of the sign of $t_{a}$, the wave functions
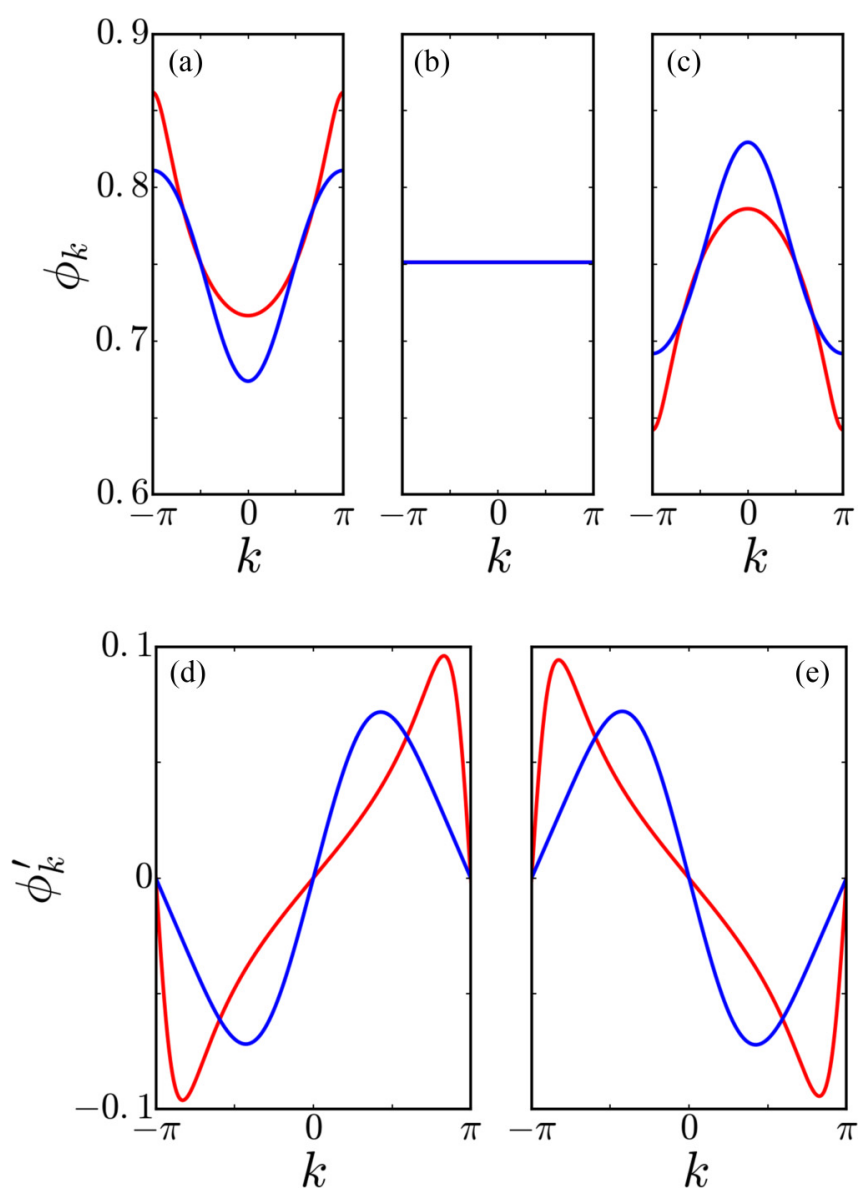

FIG. 13. Top: The ES of the SSH model with SOC in the presence of second-NN hoppings, obtained by tracing the sublattice $\mathrm{B}$ out, for different $t_{a}$. (a) $t_{a}=-0.5$, (b) $t_{a}=0$, and (c) $t_{a}=0.5$. Bottom: The entanglement band velocity at (d) $t_{a}=-0.5$ and (e) $t_{a}=0.5$.

of the in-gap states are located at opposite edges. The ES can shed some light on the role of these two different cases. A useful cut is obtained by tracing out the $b$ sites, which leaves us with a chain of $a$ sites. The reduced correlation matrix in momentum space is

$$
G=\left(\begin{array}{cc}
\left\langle a_{k \uparrow}^{\dagger} a_{k \uparrow}\right\rangle & \left\langle a_{k \uparrow}^{\dagger} a_{k \downarrow}\right\rangle \\
\left\langle a_{k \downarrow}^{\dagger} a_{k \uparrow}\right\rangle & \left\langle a_{k \downarrow}^{\dagger} a_{k \downarrow}\right\rangle
\end{array}\right) .
$$

Its eigenvalues $\eta_{k}^{(1)}$ and $\eta_{k}^{(2)}$ lead to the ES:

$$
\phi_{k}^{(i)}=1-2 \arctan \eta_{k}^{(i)}, \quad i=1,2 .
$$

Figure 13 shows that the ES depends sensitively on $t_{a}$. For vanishing $t_{a}$ the ES is dispersionless. We have verified that it is true for all SPT phases of Fig. 1. For finite $t_{a}$, the ES is switched upside down if $t_{a}$ is replaced by $-t_{a}$. This behavior is well illustrated in terms of the entanglement band velocity, the group velocity of the ES (lower part of Fig. 13), which changes sign if $t_{a}$ is replaced by $-t_{a}$. The entanglement band velocity is thus a valid quantity both for distinguishing $C P$ from SPT phases and for telling the two $C P$ phases from each other. 
We now show that the Zak phase can also be used for separating the various phases. For simplicity, we neglect both SOC and third-neighbor hopping. Thus the Bloch Hamiltonian is a $2 \times 2$ matrix as in Eq. (25) with $\Delta$ replaced by $\omega_{k}=2 t_{a} \cos k$ and with $1+\delta+(1-\delta) e^{\mp i k}$ as nondiagonal elements. We choose a complex representation as in Eq. (25) and find

$$
\Phi=-\frac{1-\delta}{2} \int_{-\pi}^{\pi} d k \frac{\omega_{k}}{E_{k}} \frac{1-\delta+(1+\delta) \cos k}{E_{k}^{2}},
$$

where $E_{k}=\sqrt{\omega_{k}^{2}+2\left[1+\delta^{2}+\left(1-\delta^{2}\right) \cos k\right]}$. The Zak phase vanishes for $t_{a} \rightarrow 0$, as expected, but it also vanishes for $\delta \rightarrow \pm 1$, where the system becomes a two-leg ladder with hopping $\pm t_{a}$ along the legs and 2 on the rungs. Moreover, $\Phi$ changes signs if $t_{a}$ is replaced by $-t_{a}$.

Is there a physical quantity which is proportional to $\Phi$, as in the charge density wave case? Proceeding as in Sec. III B we can easily calculate correlation functions. The density is homogeneous, but the bond strengths $\left\langle a_{n \sigma}^{\dagger} a_{n+1 \sigma}\right\rangle$ and $\left\langle b_{n \sigma}^{\dagger} b_{n+1 \sigma}\right\rangle$ are different and depend on $t_{a}$. We find

$$
\left\langle b_{n \sigma}^{\dagger} b_{n+1 \sigma}\right\rangle-\left\langle a_{n \sigma}^{\dagger} a_{n+1 \sigma}\right\rangle=\frac{1}{2 \pi} \int_{-\pi}^{\pi} d k \frac{\omega_{k} \cos k}{E_{k}} .
$$

This quantity is somewhat different from the Zak phase, Eq. (36), but for small values of $t_{a}$ both expressions vary linearly with $t_{a}$ and tend to zero for $t_{a} \rightarrow 0$. A similar asymmetry is found if the ES, obtained by tracing out the $b$ sites, is compared with the ES calculated by tracing out the $a$ sites. For a fixed value of $t_{a}$ the corresponding spectra are precisely those of the left and right parts of Fig. 13, respectively.

\section{SUMMARY AND CONCLUDING REMARKS}

In this paper, we have investigated a generalized SSH model, which embodies bond alternation, SOC, and hopping beyond nearest-neighbor sites. Depending on its parameters, the model produces a rich variety of features, partly topological, partly nontopological. Two main cases have been considered. On the one hand, we have studied the model where hopping is only allowed to occur between different sublattices (between odd and even sites). The Hamiltonian then has the symmetries of charge conjugation, time reversal, and chirality. This system shows a rich phase diagram with the well-known properties of topological insulators, including quantized topological invariants, symmetry-protected edge states, and topological phase transitions. On the other hand, we have studied the effects of next-nearest-neighbor hopping (hopping within sublattices), which destroys chargeconjugation (and therefore also chiral) symmetry. This system has a topologically trivial ground state. Nevertheless, some signatures of topologically nontrivial states can remain intact, such as integer winding numbers or robust edge states, especially if the additional hopping term is weak.

We have used various tools to characterize the different ground states. Entanglement, a concept of central importance in quantum information theory, has been very useful in our study. Specific quantities such as ES, entanglement entropy, and entanglement gap have been determined and shown to provide detailed information about topological transitions in the chirally symmetric case, but also about the (nontopological) ground states in the absence of chiral symmetry. In the particular case where both charge-conjugation symmetry $C$ and parity $P$ are broken, but $C P$ is conserved (this happens if the two hopping parameters between next-nearest-neighbor sites have the same magnitude but different signs) we have introduced an "entanglement band velocity" to distinguish this case from the topologically nontrivial case, where both $C$ and $P$ are conserved.

The Zak phase (the Berry phase for a one-dimensional Bloch band) has been a versatile tool both for determining the winding numbers of different topological states and as a measure for parity-symmetry breaking. We have used the fact that the Zak phase is not gauge invariant by choosing different specific gauges in these two cases. For topologically nontrivial states, a mixed real-complex representation for the eigenfunctions yields winding numbers in one-to-one correspondence with the numbers of edge states (bulk-boundary correspondence), while a fully complex representation is appropriate for describing states with broken parity, because in this case the Zak phase is proportional to a physical quantity measuring the amount of symmetry breaking (the polarization in the closely related Aubry-André model).

The specific case of broken chirality and conserved parity (for nonalternating next-nearest-neighbor hopping) also shows interesting and partly unexpected features. The Zak phase remains quantized for gapped states, although these are topologically trivial. At the same time, some topological transitions (occurring in the absence of next-nearest-neighbor hopping) are broadened into metallic regions, due to overlaps between conduction and valence bands.

\section{ACKNOWLEDGMENTS}

The authors would like to thank Mahsa Seyedheydari for her help at the initial stage of this paper. N.A. thanks Henrik Johannesson for useful discussions.

\section{APPENDIX: FROM AA TO SSH}

Here we show that a simple unitary transformation can eliminate the on-site term of the Hamiltonian Eq. (2). We consider the case of periodic boundary conditions and introduce fermionic operators $\alpha_{i \sigma}, \beta_{i \sigma}$ through the Bogolyubov transformation:

$$
\begin{aligned}
& a_{i \sigma}=\cos \vartheta \alpha_{i \sigma}+\sin \vartheta \beta_{i \sigma} \\
& b_{i \sigma}=-\sin \vartheta \alpha_{i \sigma}+\cos \vartheta \beta_{i \sigma}
\end{aligned}
$$

Inserting these equations into Eq. (2) and requiring that there be no alternating on-site term in the representation, we obtain the condition $\tan \vartheta=\Delta$ (we put $t=1$ ). With $\cos \vartheta=\frac{1}{\tau}$, $\sin \vartheta=\frac{\Delta}{\tau}$, where $\tau:=\sqrt{1+\Delta^{2}}$, we find

$$
\begin{aligned}
H_{\mathrm{AA}}= & \sum_{i \sigma}\left\{\tau\left(\alpha_{i \sigma}^{\dagger} \beta_{i \sigma}+\text { H.c. }\right)+\frac{1+\tau}{2 \tau}\left(\alpha_{i+1 \sigma}^{\dagger} \beta_{i \sigma}+\text { H.c. }\right)\right. \\
& -\frac{\Delta}{2 \tau}\left(\alpha_{i \sigma}^{\dagger} \alpha_{i+1 \sigma}-\beta_{i \sigma}^{\dagger} \beta_{i+1 \sigma}+\text { H.c. }\right) \\
& \left.+\frac{1-\tau}{\tau}\left(\alpha_{i \sigma}^{\dagger} \beta_{i+1 \sigma}+\text { H.c. }\right)\right\}
\end{aligned}
$$


The various terms represent nearest-neighbor hopping (line 1), second-neighbor hopping with alternating sign (line 2), and third-neighbor hopping (only on half of the possible bonds).
[1] K. V. Klitzing, G. Dorda, and M. Pepper, Phys. Rev. Lett. 45, 494 (1980).

[2] D. C. Tsui, H. L. Stormer, and A. C. Gossard, Phys. Rev. Lett. 48, 1559 (1982).

[3] S. Rachel, Rep. Prog. Phys. 81, 116501 (2018).

[4] M. Z. Hasan and C. L. Kane, Rev. Mod. Phys. 82, 3045 (2010).

[5] K. L. Hur, L. Henriet, A. Petrescu, K. Plekhanov, G. Roux, and M. Schiró, C. R. Phys. 17, 808 (2016).

[6] V. Galitski and I. B. Spielman, Nature 494, 49 (2013).

[7] Z. Gong, Y. Ashida, K. Kawabata, K. Takasan, S. Higashikawa, and M. Ueda, Phys. Rev. X 8, 031079 (2018).

[8] C.-K. Chiu, J. C. Y. Teo, A. P. Schnyder, and S. Ryu, Rev. Mod. Phys. 88, 035005 (2016).

[9] J. Zak, Phys. Rev. Lett. 62, 2747 (1989).

[10] M. A. Nielsen and I. L. Chuang, Quantum Computation and Quantum Information (Cambridge University Press, Cambridge, 2000).

[11] A. Osterloh, L. Amico, G. Falci, and R. Fazio, Nature 416, 608 (2002).

[12] A. Kitaev and J. Preskill, Phys. Rev. Lett. 96, 110404 (2006).

[13] H. Li and F. D. M. Haldane, Phys. Rev. Lett. 101, 010504 (2008).

[14] A. M. Läuchli, E. J. Bergholtz, J. Suorsa, and M. Haque, Phys. Rev. Lett. 104, 156404 (2010).

[15] A. Sterdyniak, A. Chandran, N. Regnault, B. A. Bernevig, and P. Bonderson, Phys. Rev. B 85, 125308 (2012).

[16] M. Pouranvari and J. Abouie, Phys. Rev. B 100, 195109 (2019).

[17] F. Pollmann, A. M. Turner, E. Berg, and M. Oshikawa, Phys. Rev. B 81, 064439 (2010).

[18] D. Poilblanc, Phys. Rev. Lett. 105, 077202 (2010).

[19] R. Thomale, D. P. Arovas, and B. A. Bernevig, Phys. Rev. Lett. 105, 116805 (2010).

[20] F. Pollmann, E. Berg, A. M. Turner, and M. Oshikawa, Phys. Rev. B 85, 075125 (2012).

[21] H. Yao and X.-L. Qi, Phys. Rev. Lett. 105, 080501 (2010).

[22] O. Gauthé and D. Poilblanc, Phys. Rev. B 96, 121115(R) (2017).

[23] L. Fidkowski, Phys. Rev. Lett. 104, 130502 (2010).

[24] M. Legner and T. Neupert, Phys. Rev. B 88, 115114 (2013).

[25] A. M. Läuchli and J. Schliemann, Phys. Rev. B 85, 054403 (2012).

[26] J. Schliemann, New J. Phys. 15, 053017 (2013).

[27] Z. Moradi and J. Abouie, J. Stat. Mech. (2016) 113101.

[28] W. P. Su, J. R. Schrieffer, and A. J. Heeger, Phys. Rev. Lett. 42, 1698 (1979).

[29] R. Jackiw and C. Rebbi, Phys. Rev. D 13, 3398 (1976).

[30] J. Pople and S. Walmsley, Mol. Phys. 5, 15 (1962).

[31] A. J. Heeger, S. Kivelson, J. R. Schrieffer, and W. P. Su, Rev. Mod. Phys. 60, 781 (1988).

[32] P. Delplace, D. Ullmo, and G. Montambaux, Phys. Rev. B 84, 195452 (2011)
[33] M. Atala, M. Aidelsburger, J. T. Barreiro, D. Abanin, T. Kitagawa, E. Demler, and I. Bloch, Nat. Phys. 9, 795 (2013).

[34] N. R. Cooper, J. Dalibard, and I. B. Spielman, Rev. Mod. Phys. 91, 015005 (2019).

[35] T. Holstein, Ann. Phys. (NY) 8, 325 (1959).

[36] S. Aubry and G. André, Ann. Israel Phys. Soc. 3, 18 (1980).

[37] M. J. Rice and E. J. Mele, Phys. Rev. Lett. 49, 1455 (1982).

[38] L. Li, Z. Xu, and S. Chen, Phys. Rev. B 89, 085111 (2014).

[39] B. Pérez-González, M. Bello, A. Gómez-León, and G. Platero, Phys. Rev. B 99, 035146 (2019).

[40] A. Altland and M. R. Zirnbauer, Phys. Rev. B 55, 1142 (1997).

[41] J. I. Väyrynen and T. Ojanen, Phys. Rev. Lett. 107, 166804 (2011).

[42] Z. Yan and S. Wan, Europhys. Lett. 107, 47007 (2014).

[43] M. Bahari and M. V. Hosseini, Phys. Rev. B 94, 125119 (2016).

[44] Y. Yao, M. Sato, T. Nakamura, N. Furukawa, and M. Oshikawa, Phys. Rev. B 96, 205424 (2017).

[45] C. E. Whittaker, E. Cancellieri, P. M. Walker, B. Royall, L. E. Tapia Rodriguez, E. Clarke, D. M. Whittaker, H. Schomerus, M. S. Skolnick, and D. N. Krizhanovskii, Phys. Rev. B 99, 081402(R) (2019).

[46] S. Ryu, A. P. Schnyder, A. Furusaki, and A. W. W. Ludwig, New J. Phys. 12, 065010 (2010).

[47] M. V. Berry, Proc. R. Soc. London A 392, 45 (1984).

[48] L. Fu and C. L. Kane, Phys. Rev. B 76, 045302 (2007).

[49] M. Pletyukhov, D. M. Kennes, J. Klinovaja, D. Loss, and H. Schoeller, Phys. Rev. B 101, 161106(R) (2020).

[50] N. Laflorencie, Phys. Rep. 646, 1 (2016).

[51] M.-C. Chung and I. Peschel, Phys. Rev. B 64, 064412 (2001).

[52] S.-A. Cheong and C. L. Henley, Phys. Rev. B 69, 075111 (2004).

[53] I. Peschel and V. Eisler, J. Phys. A 42, 504003 (2009).

[54] A. Alexandradinata, T. L. Hughes, and B. A. Bernevig, Phys. Rev. B 84, 195103 (2011).

[55] S. Ryu and Y. Hatsugai, Phys. Rev. B 73, 245115 (2006).

[56] A. F. Albuquerque, F. Alet, C. Sire, and S. Capponi, Phys. Rev. B 81, 064418 (2010).

[57] X. Luo, K. Zhou, W. Liu, Z. Liang, and Z. Zhang, Phys. Rev. A 89, 043612 (2014).

[58] J. Sirker, M. Maiti, N. P. Konstantinidis, and N. Sedlmayr, J. Stat. Mech. (2014) P10032.

[59] E. J. König, A. Levchenko, and N. Sedlmayr, Phys. Rev. B 93, 235160 (2016).

[60] S. Rufo, N. Lopes, M. A. Continentino, and M. A. R. Griffith, Phys. Rev. B 100, 195432 (2019).

[61] W. Chen, M. Legner, A. Rüegg, and M. Sigrist, Phys. Rev. B 95, 075116 (2017).

[62] W. Chen and A. P. Schnyder, New J. Phys. 21, 073003 (2019).

[63] M. Henkel, Conformal Invariance and Critical Phenomena (Springer-Verlag, Berlin, 1999). 
[64] C. Itzykson and J.-M. Drouffe, Statistical Field Theory (Cambridge University Press, Cambridge, 1989).

[65] G. Vidal, J. I. Latorre, E. Rico, and A. Kitaev, Phys. Rev. Lett. 90, 227902 (2003).

[66] X.-L. Qi, T. L. Hughes, and S.-C. Zhang, Phys. Rev. B 78, 195424 (2008).
[67] A. P. Schnyder, S. Ryu, A. Furusaki, and A. W. W. Ludwig, Phys. Rev. B 78, 195125 (2008).

[68] R. D. King-Smith and D. Vanderbilt, Phys. Rev. B 47, 1651 (1993).

[69] R. Resta, Rev. Mod. Phys. 66, 899 (1994).

[70] C.-T. Hsieh, T. Morimoto, and S. Ryu, Phys. Rev. B 90, 245111 (2014). 